\title{
THICK DATA ANALYTICS: A HUMAN CENTRIC APPROACH FOR UNDERSTANDING CONSUMER INSIGHTS
}

\author{
Sabah Mohammed ${ }^{1}$, Jinan Fiaidhi ${ }^{2}$ and Sami Mohammed ${ }^{3 *}$ \\ ${ }^{1}$ Department of Computer Science, Lakehead University, Canada \\ ${ }^{2}$ Department of Computer Science, Lakehead University, Canada \\ ${ }^{3}$ Department of Computer Science, University of Victoria, Canada \\ ${ }^{1}$ sabah.mohammed@lakeheadu.ca, ${ }^{2}$ jfiaidhi@lakeheadu.ca, ${ }^{3}$ smohamm@uvic.ca
}

Abstract - Gartner predicts that in 2017 that $60 \%$ of all big data projects fail. As bad as that sounds, the reality is actually worse. The big data projects failed because of the obsession with quantitative data at the expense of qualitative consumer centric data. Thick data puts more weight on the qualitative data to provide the context that enables the business to understand consumer insights. Based on thick data analytics, one can understand the quirks of human behavior to predict how an individual's relationship with the business service or product will evolve over time. Without this understanding, quantitative patterns that the business may uncover that suggest people will behave in a certain way could be based in a world that no longer exists. This paper highlights the main approaches that any business can use to uncover thick data in order to understand the consumer's insights.

\section{Keywords - Thick Data, Consumer Insights, Human Centric Approaches}

\section{INTRODUCTION}

Majority of customers in every business are now online and they leave a trail of data on social media sites, blogs and portals, messages of all types and lots of traces on search engines. This data provides valuable customer insights that businesses should be analyzing in order to "know their customers". A business who know their customers can make smarter decisions that maximize consumer loyalty without spending money on unnecessary exercises (e.g. general advertising, rebates, discounts and special offers). The general believe has been always to rely on Big Data and predictive analytics to make relevant, personalized, and precisely timed offers to customers. However, using such approach proves to be daunting if it is built on prediction models that are based on quantitative datasets and do not incorporate important consumer values and insights. According to Tricia Wang [1] "What is measurable isn't the same as what is valuable". There are many alarming examples that proves the failure of using pure quantitative consumer data to derive business decisions like the Nokia failure although Nokia have collected huge amount of data about their customers and the market but failed to alert the company about the consumer's values and the market trends for the smartphone technology [2]. Figure 1 illustrate the smartphone market sales between 2007 and 2011 where it shows the failure of Nokia with its Symbian smartphone compared to the other venders smartphones.

Received: December 29, 2018

Reviewed: February 5, 2019

Accepted: February 15, 2019

* Corresponding Author 


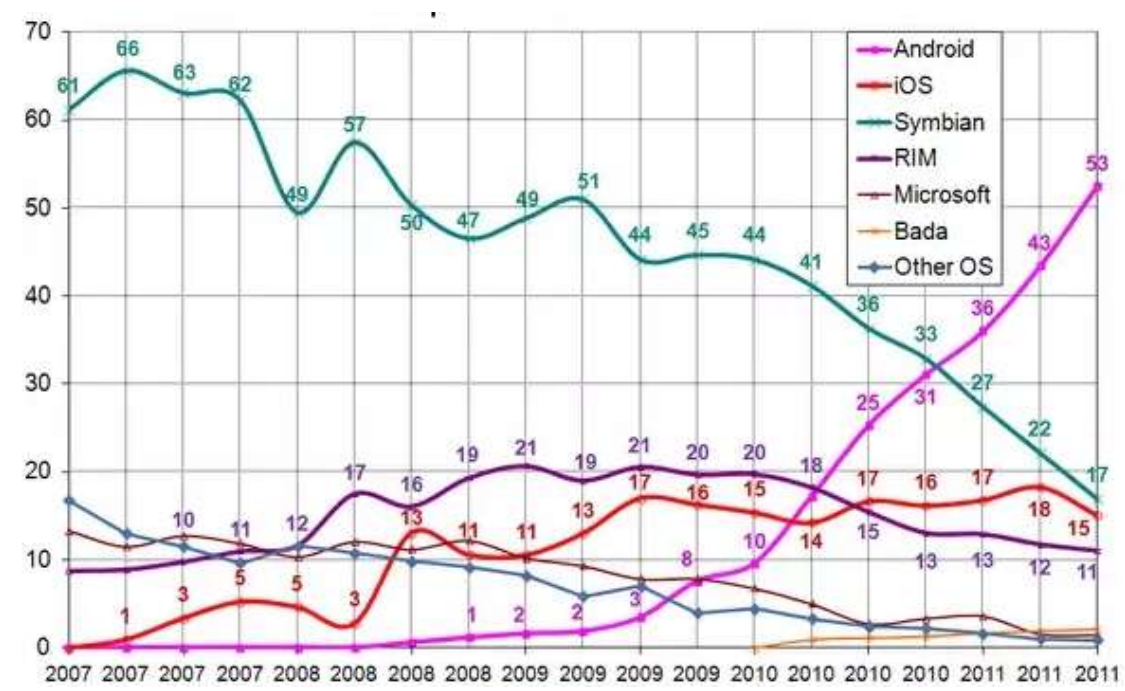

Fig. 1 The Smartphone Sales during the period of 2007-2011.

Many missing factors are associated with using general quantitative data to tap on consumer insights. It includes the power to understand the nuances of consumer behavior with the best-in-class consumer insights technologies. It also includes techniques to provide answers to some of the consumer centric questions like:

$>$ How consumers think and feel about different alternatives (brands, products, services, and retailers)

> How consumers reason and select between different alternatives

$>$ What is the behavior of consumers while researching and shopping

$>$ How consumer behavior is influenced by their environment (peers, culture, media)

$>$ How to navigate for insights when the consumer data are in doubt or associated with uncertainty due to data inconsistency and incompleteness, ambiguities, latency, deception, model approximations, low accuracy, low quality, unclear truthfulness or with lower trustworthiness

$>$ How to navigate for insights when consumer data continuously changes through the use of the differing ways of data interpretation

$>$ What are the relative importance of different complex consumer data from different perspectives and locations?

Answering these questions will enable us to understand the demographics, interests, and preferences of the customers. What we know about consumer behavior is only the general consideration that it is influenced by three factors [3]:

Personal factors - A person's interests and opinions. These will be affected by demographics such as age, gender, culture, profession, background and so on.

Psychological factors - Everybody's response to a particular marketing campaign will be based on their perceptions and attitudes. A person's ability to comprehend information, their perception of their need, their attitude, will all play a part.

Social factors - Peer groups, from family and friends to social media influence. This factor also includes social class, income, and education level. 
What we know also is that consumers want convenience, choice and control when they shop.

\section{THE THICK DATA APPROACH}

From the above discussion we know that big data is not working. The industry today is worth in excess of $\$ 122$ billion, yet Gartner predicts that in $201760 \%$ of all big data projects fail [4]. As bad as that sounds, the reality is actually worse. According to Gartner analyst Nick Heudecker, Gartner was "too conservative" with its $60 \%$ estimate. The real failure rate is "[C]loser to 85 percent." [5] Actually, Big data is generated by the millions of touchpoints that organizations have with customers. It provides quantitative information from which analysis can reveal general insights that lead to new efficiencies and improved processes. For example, traffic data and predictive models have enabled airlines to precisely match aircraft capacity with seat demand while slashing their service on routes that rarely reach capacity. Planes now average about $83 \%$ full as a result. This is fairly easy to do simply by looking at historical patterns of behavior, yet while quantitative analysis can achieve results when it comes to these examples, if you are trying to use data to better understand customer behavior and trends for marketing particularly, as many are, more information is needed. You need to understand the human emotion behind the numbers. This is perhaps best evidenced by polling errors in last year's EU referendum and US presidential elections, where the absence of public sentiment in the majority of statistical models meant that the majority got it dramatically wrong [6]. This is why everyone currently singling out the major contributing factor to the failure of all the big data projects to the obsession with quantitative data at the expense of qualitative data. Organizations are essentially marginalizing the human element and context that must accompany numbers for them to be worth anything.

Thick data a concept coined by Tricia Wang puts more weight on the qualitative data to provide the context that enables the business to understand consumer insights. Based on Thick data Analytics, we can understand the quirks of human behavior to predict how an individual's relationship with the business service or product will evolve over time. Without this understanding, quantitative patterns that the business may uncover that suggest people will behave in a certain way could be based in a world that no longer exists. Thick data analytics may use any of the following techniques to tap on consumer insights [3]:

- Customer Reviews and Complaints - Reading customer reviews can highlight common problems or wishes. Customer feedback is an opportunity to make your business better. Each individual customer complaint potentially represents a much larger sentiment. Therefore, it's worthwhile to understand your customers' experience and identify areas for improvement for your business.

- Q\&A sites - These sites can give you an idea of the questions and concerns that people have in relation to your brand, service or product.

- Surveys - Online surveys can be easily set up with sites like Survey Monkey and allow you to ask specific questions.

- Focus groups - Bring a group of consumers together and ask them questions directly.

- Keyword research - A mainstay of SEO, keyword research can tell you what consumers are interested in and the relative level of interest. It also helps to reveal the language they are using.

- Google Analytics - Analytics can be used to tell you where your traffic is coming from. The Audience tab shows geography, interests, and a range of demographics. Google analytics can help you understand customer behavior 
- Competitor analysis - This can provide useful information about consumers that are shopping in your vertical but don't buy from your brand. Read our post on the top competitor analysis tools.

- Blog comments - Comments on your blog can be a good way of discovering any questions your audience might have.

- Twitter Insiders - Twitter recently launched Insiders, a 12,000 strong focus group of US \& UK Twitter users.

- Google trends - Google Trends can help you to understand if a topic is becoming more or less popular.

- Government data - Government data is available for free and can help you understand a group, and several other sources can also be accessed without charge.

- Social media - Millions of people reflect their lives on social media, so information that can enrich several strands of consumer behavior can be uncovered with the right tools.

However, finding the hidden value in customer feedback for example is exceptionally complicated, and it is still too early to leave it to machine learning as the sample size is quite small (on average only $4 \%$ of unsatisfied customers in any business). Indeed, the lack of customer feedback doesn't necessarily mean your customers are completely satisfied with your business. If we rely solely on big data, we end up with a warped sense of the world in which human beings are simply numbers to be fed into an algorithm. This is not to say that it is useless, nor that in many cases it can be used alone. It is still a powerful and helpful tool that companies should invest in. However, companies should also invest in gathering and analyzing thick data to uncover the deeper, more human meaning behind big data. For the consumer feedback the business need to start using techniques from the thick data to find the hidden value of a complaint, we'll need to start by collecting all of the relevant information, such as:

○ Details of incident

- Date

- Location

○ Product

- Customer service agent involved

Next, we'll want to visualize the data to reveal insights such as trends and patterns even on small collected sample. The business may want to consider visualizing many factors related to consumer feedback and complaints like:

- Volume and nature of customer feedback

- Customer attrition

- Support call volume

- Warranty claims

- Number of complaints over time

- Number of cancellations

After you've established this thick data information, you can look at the most important details that it contains. This will be the sort of data that will help the business to really improve their interactions with customers. It's possible to see the number of errors 
made, how many people hung up, how many products returned and how many calls are being handled per minute, for example. This all helps to boost the customers' satisfaction and that's the key to avoiding further complaints.

\section{CONCLUSIONS}

Big data helps business to quantify the world while Thick data helps the business to explain why people do what they do. By taking time to watch and interact with people will give the business the insights into the way consumers think and behave and, crucially, what they might do next. Figure 2 illustrate the role of Thick Data in understanding consumer's insights. It also states the role of Big Data analytics to follow afterwards.

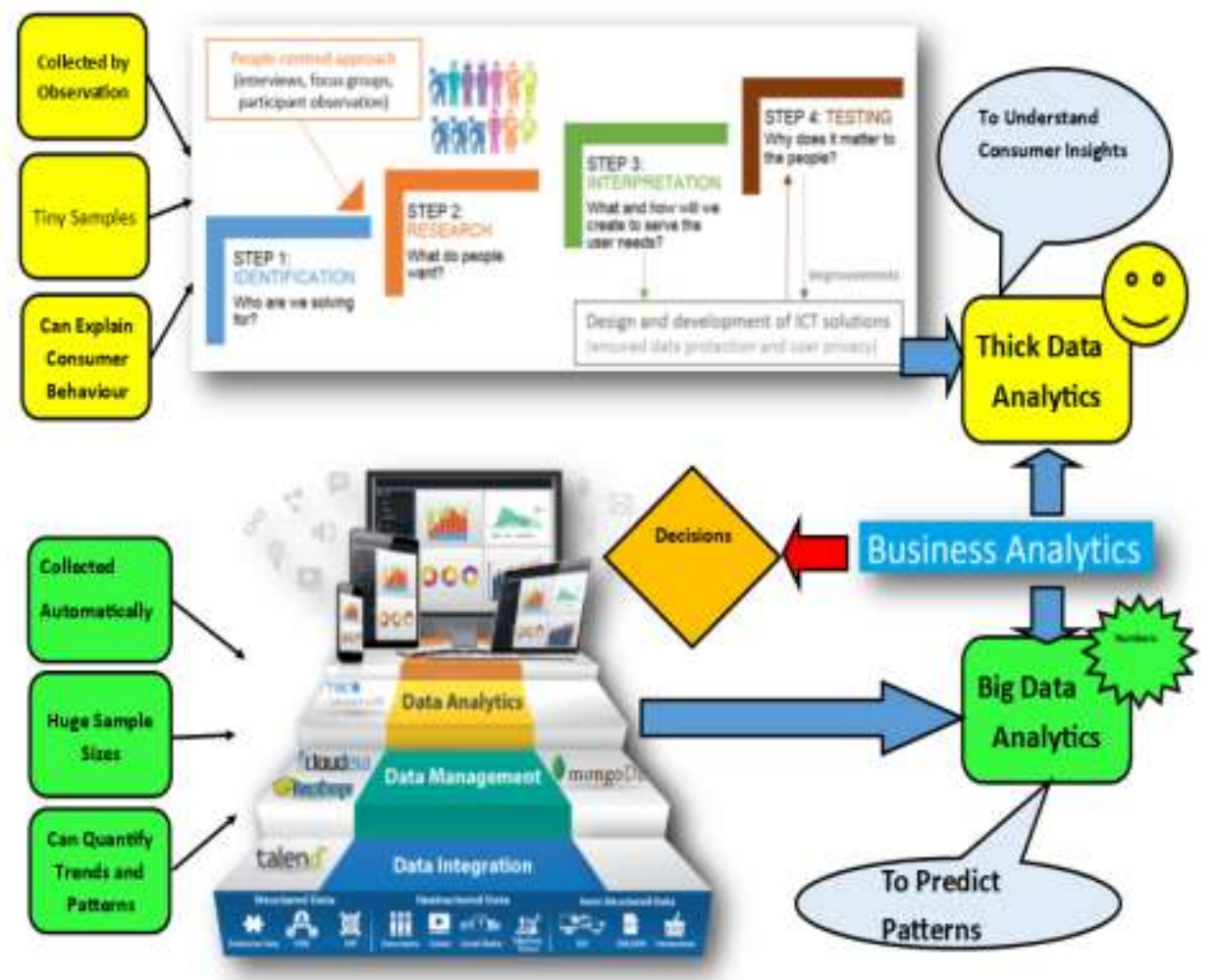

Fig. 2 Thick Data Starts First

The authors are currently involved in performing major Thick Data analytics based on the NY Open Data ${ }^{1}$ that include many consumer complaints about businesses at this city. More findings will follow this summary paper.

\section{REFERENCES}

[1] Wang, Tricia, Why Big Data Needs Thick Data, Medium.com blog, Jan 20, 2016, Available Online: https://medium.com/ethnography-matters/why-big-data-needs-thick-data-b4b3e75e3d7

[2] BRAND MINDS, Why did Nokia fail and what can you learn from it? Jul 24, 2018, Available online: https://medium.com/multiplier-magazine/why-did-nokia-fail-81110d981787

[3] Kit Smith, How to Understand and Influence Consumer Behavior, Brand Watch, October 28th 2016, Available Online: https://www.brandwatch.com/blog/how-understand-influence-consumer-behavior/

[4] Laurence Goasduff, Taking a First Step to Advanced Analytics, Garthner, September 15, 2015, Available online: https://www.gartner.com/smarterwithgartner/taking-a-first-step-to-advancedanalytics/

\footnotetext{
${ }^{1}$ https://data.cityofnewyork.us/browse?category=Business
} 
International Journal of Control and Automation

Vol. 12, No. 2 (2019)

[5] Matt Asay By Matt Asay, Tech Republic, November 10, 2017, in Big Data on November 10, 2017, Available Online: https://www.techrepublic.com/article/85-of-big-data-projects-fail-but-yourdevelopers-can-help-yours-succeed/

[6] James Ovenden, Why Big Data Needs Thick Data, Innovation Channels, 2018, Available Online: https://channels.theinnovationenterprise.com/articles/why-big-data-needs-thick-data 